\title{
The challenge of imaging-guided therapeutic management
}

\author{
Leslee J. Shaw, PhD, FASNC, FACC, FAHA
}

See related article, pp. 1021-1025

In this issue of the journal, Bove et $\mathrm{al}^{1}$ challenge the longstanding belief that imaging is required to guide prevention goals and to target the intensity of therapeutic intervention. For many years, the tenet of ischemia-guided management has been the overarching strategy for management of symptomatic patients. ${ }^{2}$ Additionally, for primary prevention, an aggregate of laboratory and clinical measurements integrated into a global risk score, such as the Framingham risk score, is the standard upon which targeted lipid and hypertensive management are based. ${ }^{3}$ More recently, imaging of subclinical atherosclerosis has been shown to provide more direct evidence of the extent and severity of cardiovascular disease (CVD) and to provide data on longterm prognosis. ${ }^{3}$ A synthesis of evidence was recently published in a recent guideline from the American College of Cardiology Foundation/American Heart Association noting that several imaging tests provide substantive information to uniquely detect risk above and beyond the Framingham risk score. ${ }^{3}$ This guideline puts forth recommendations for the utility of coronary artery calcium scoring and other CVD screening tests. In general, this guideline reports that (1) direct imaging of the vasculature, such as with coronary calcium scoring, can improve risk detection and (2) the use of any global risk score is decidedly less accurate in large segments of the adult population including women, younger men, non-US cohorts, and individuals of diverse racial and ethnic backgrounds.

In the current issue, Bove et al argue that nearly all of patients who presented for an outpatient visit could be managed without the need for any further testing. They posit that good clinical judgment and an adequate

From the Emory University School of Medicine, Atlanta, GA. Reprint requests: Leslee J. Shaw, PhD, FASNC, FACC, FAHA, Emory

University School of Medicine, Atlanta, GA; lshaw3@emory.edu. J Nucl Cardiol 2011;18:995-6.

$1071-3581 / \$ 34.00$

Copyright (c) 2011 American Society of Nuclear Cardiology.

doi:10.1007/s12350-011-9440-6 evaluation of cardiac risk factors can be used to target ensuing therapeutic management. By applying their reasoning, a patient presenting for evaluation of hypertension with suboptimal control could then be targeted for intensification of therapy or for switching to an alternative medication without any guidance from additional novel imaging or laboratory biomarkers. This is certainly true that adequate control of traditional cardiac risk factors should be guided by observed measurements of blood pressure, glucose, and lipids; with ensuing therapeutic intervention titrated to adequate control based on established targets. The authors note an extremely important part of a patient's visit- to assess and identify areas of clinical need.

However, this report notes only one step in the care of a patient; that of immediate attention to ongoing risk factor control. But, what about concerns about the patient's long-term risk and targeting strategies for further reductions in CVD morbidity and mortality. This is where the recent guidelines on detection of asymptomatic high risk individuals has targeted a secondary evaluation of the patient's underlying burden of subclinical atherosclerosis with tests such as coronary artery calcium or high sensitivity C-reactive protein. ${ }^{3}$ From this guideline, the goal of this approach is to detect an asymptomatic individual who is at high long-term risk of major adverse cardiovascular events. This patient would then be targeted to secondary prevention goals (instead of primary prevention targets) as a means to reduce their high risk status and expected elevated rate of morbid and fatal complications. Thus, imaging or any CVD screening tests is used to identify high risk patients and then to initiate more intensive therapeutic management and lifestyle modifications; consistent with secondary prevention goals of a patient with established CVD.

One message from the Bove article is that we importantly need additional clinical research evidence on the impact of imaging-guided patient management on improved patient outcomes. This level of evidence derived from high quality randomized trials or registries that a coronary artery calcium scan, for example, provides important information to target effective therapeutic intervention is currently not available. The recent guideline from the American College of Cardiology/American Heart Association identified that a risk 
marker should not only provide improved, novel risk detection but also evidence of guided therapeutic benefit. $^{3}$ For all but high sensitivity C-reactive protein, guided therapeutic intervention evidence is not available. This evidence of a therapeutic benefit in terms of reduction in major cardiovascular events based on identification of high risk individuals based on high sensitivity C-reactive protein testing was recently reported from the Justification for the Use of Statins in Prevention: An Intervention Trial Evaluating Rosuvastatin (JUPITER) trial. ${ }^{4}$ In JUPITER, a total of 17,802 apparently healthy individuals with a high sensitivity C-reactive protein value of $\geq 2.0 \mathrm{mg} / \mathrm{L}$ were randomized and the results revealed that statin therapy significantly reduced the incidence of major cardiovascular events when compared to placebo (hazard ratio $=0.56 ; 95 \%$ confidence interval: $0.46-0.69, P<.00001$ ).

For coronary artery calcium scoring, similar data on targeted therapeutic intervention from a sufficiently powered clinical trial is not available. Moreover, all 4 (small) trials that used statin therapy to slow progression of coronary artery calcification were negative. ${ }^{5}$ In the St. Francis Heart Study ${ }^{6}$ stain treatment failed to reduce the primary endpoint of CVD events $(P=.08)$. Only in a (prespecified) subset of patients with a calcium score $>400$, statins reduced the incidence of CVD events by $42 \%(P=.046)$; although it is likely that this secondary analysis remained underpowered. Most of the available evidence with coronary calcium scoring notes improved adherence $^{7}$ and improved Framingham risk scores $^{8}$ when compared to patients not undergoing scanning. Thus, the extent to which the evidence lacks robustness with regards to a therapeutic risk reduction targeted by coronary calcium, Bove and colleagues ${ }^{1}$ are correct in questioning the current approach of CVD screening to guide detection and treatment of high risk individuals. Definitive trials with coronary calcium scoring are under evaluation and may provide a better evidence base upon which to guide additional screening and prevention strategies.

Yet, in this era of evidence-based medicine being applied to shape healthcare policy and coverage decisions, the fact that we do not have a national coverage decision on screening beyond a lipid panel is indefensible and remains a hurdle to further reductions in CVD morbidity and mortality. Expanded screening to include testing for subclinical atherosclerosis would require a legislative mandate but it appears that much of the evidence is of similar magnitude and quality to that of such covered services for breast cancer screening. It would seem that the evidence is coalescing and it is the time to start discussions as to how we can further avert the number one killer of Americans and patients worldwide. The general tenet of CVD screening is that early intervention results in a cost offset by reducing expensive, secondary prevention care. Thus, within our current reimbursement system, any expanded services may only be supported if they do not add costs (i.e., a "zero-sum gain"). It remains likely that the reductions in CVD events noted within several trials as well as improvements in the Framingham risk score could provide significant cost savings to the healthcare system while importantly ameliorating the burden of symptomatic, costly secondary prevention for many asymptomatic high risk individuals.

\section{References}

1. Bove AA, Santamore WP, Homko C, Kashem A, Cross R, McConnell TR, et al. Treatment of patients with intermediate cardiovascular risk: are clinical measures enough? J Nucl Cardiol 2011. doi:10.1007/s12350-011-9415-7.

2. Califf RM, Armstrong PW, Carver JR, D'Agostino RB, Strauss WE. 27th Bethesda Conference: matching the intensity of risk factor management with the hazard for coronary disease events. Task Force 5. Stratification of patients into high, medium and low risk subgroups for purposes of risk factor management. J Am Coll Cardiol 1996;27:1007-19.

3. Greenland P, Alpert JS, Beller GA, et al. 2010 ACCF/AHA guideline for assessment of cardiovascular risk in asymptomatic adults: A report of the American College of Cardiology Foundation/ American Heart Association Task Force on Practice Guidelines. J Am Coll Cardiol 2010;56:e50-103.

4. Ridker PM, Danielson E, Fonseca FA, et al. Rosuvastatin to prevent vascular events in men and women with elevated C-reactive protein. N Engl J Med 2008;359:2195-207.

5. Oudkerk M, Stillman AE, Halliburton SS, et al. Coronary artery calcium screening: Current status and recommendations from the European Society of Cardiac Radiology and North American Society for Cardiovascular Imaging. Int $\mathbf{J}$ Cardiovasc Imaging 2008;24:645-71.

6. Arad Y, Spadaro LA, Roth M, Newstein D, Guerci AD. Treatment of asymptomatic adults with elevated coronary calcium scores with atorvastatin, vitamin C, and vitamin E: the St. Francis Heart Study randomized clinical trial. J Am Coll Cardiol 2005;46:166-72.

7. Taylor AJ, Bindeman J, Feuerstein I, et al. Community-based provision of statin and aspirin after the detection of coronary artery calcium within a community-based screening cohort. J Am Coll Cardiol 2008;51:1337-41.

8. Rozanski A, Gransar H, Shaw LJ, et al. Impact of coronary artery calcium scanning on coronary risk factors and downstream testing the EISNER (early identification of subclinical atherosclerosis by noninvasive imaging research) prospective randomized trial. J Am Coll Cardiol 2011;57:1622-32. 